\title{
Is it worth paying more for emergency hormonal contraception? The cost-effectiveness of ulipristal acetate versus levonorgestrel $1.5 \mathrm{mg}$
}

\author{
Christine M Thomas, Ramona Schmid, Sharon Cameron
}

\begin{abstract}
Background and methodology Emergency hormonal contraception $(\mathrm{EHC})$ can reduce unintended pregnancy and the associated costs and consequences for the individual and National Health Service (NHS). Levonorgestrel (LNG $1.5 \mathrm{mg}$ ) is currently the standard of care in the UK; however, it is not licensed for use $>72$ hours after unprotected sexual intercourse (UPSI). This cost-effectiveness analysis compares LNG $1.5 \mathrm{mg}$ with ulipristal acetate (UPA) (ellaOne ${ }^{\circledR}$ ), a new emergency hormonal contraceptive that is licensed for use up to 120 hours post-UPSI. The costs of both drugs and the costs of the consequences of unintended pregnancy - namely miscarriage, induced abortion and birth - are compared in a decision model from the perspective of the UK NHS.
\end{abstract}

Results The incremental cost-effectiveness ratio (ICER) is the cost of preventing one additional unintended pregnancy with UPA and is calculated to be £311 compared to LNG $1.5 \mathrm{mg}$ when taken up to 120 hours post-UPSI. In sensitivity analysis, looking at different time frames and costs, the ICER ranges from $£ 183$ to $£ 500$. All these costs are less than the estimated cost of an unintended pregnancy (£948) regardless of the outcome or the cost of an induced abortion (£672).

Discussion and conclusions Even when considering only the direct costs of an unintended pregnancy, UPA represents value for money as a method of EHC when taken up to 120 hours post-UPSI. UPA is a cost-effective alternative to LNG $1.5 \mathrm{mg}$ for all women presenting for EHC.

Keywords cost-effectiveness, emergency contraception, health economics, levonorgestrel, ulipristal acetate J Fam Plann Reprod Health Care 2010; 36(4): 197-201 (Accepted 20 July 2010)

\section{Introduction}

Contraception is regarded as a cost-effective use of health care resources compared to not using any method. ${ }^{1}$ The cost-effectiveness of emergency contraception (EC) has been studied in other countries including Canada, Australia and the USA. ${ }^{2-4}$ EC has the potential to reduce unintended pregnancy and the associated consequences for the individual and National Health Service (NHS). A Scottish study undertaken in 2005 found that $90 \%$ of pregnancies among women requesting induced abortion were unintended. ${ }^{5}$ Furthermore, the study demonstrated that up to one-third of pregnancies proceeding to birth were unintended and may be prevented by EC. In 2008, there were more than 192000 abortions performed in England, Scotland and Wales 6,7 that were funded by the NHS. Given that the average cost of an NHS abortion is $£ 672^{8}$ this equates to a cost to the NHS of more than $£ 129$ million. Until recently in the UK levonorgestrel $1.5 \mathrm{mg}$ (LNG $1.5 \mathrm{mg}$ ) was the only product available for use as emergency hormonal contraception (EHC) up to 72 hours after unprotected sexual intercourse (UPSI). Whilst women are also offered the intrauterine device (IUD) as a method of $\mathrm{EC}$, its uptake in community clinics remains at only $3 \%$ in England ${ }^{9}$ and its use is limited by its availability and need for insertion by a skilled health care professional. ${ }^{10}$ Furthermore, as IUDs provide ongoing contraceptive protection their benefits are not directly comparable with a one-time method such as EHC. There have been no UK economic assessments of EHC published. This paper presents a cost-effectiveness analysis of two EHC products: LNG $1.5 \mathrm{mg}$, which is the current standard of care in the

Tomac Consultancy Services Ltd, Cambridge, UK

Christine M Thomas, MSc, Health Economist

Laboratoire HRA Pharma, Paris, France

Ramona Schmid, PharmD, Health Economics Officer

Dean Terrace Centre, Edinburgh, UK

Sharon Cameron, MD, MRCOG, Consultant Gynaecologist

Correspondence to: Christine Thomas, Tomac Consultancy Services Ltd, 6 Shipp's Field, Waterbeach, Cambridge CB25 9DZ, UK. E-mail: christine.m.thomas@talk21.com

\section{Key message points}

- Ulipristal acetate (UPA) is a cost-effective alternative to levonorgestrel $1.5 \mathrm{mg}$ (LNG $1.5 \mathrm{mg}$ ) for women presenting up to 120 hours after unprotected sexual intercourse (UPSI).

- The cost-effectiveness of UPA compared to LNG $1.5 \mathrm{mg}$ is maintained at all time points within $0-120$ hours after UPSI.

- It is worth paying for UPA as the additional costs of preventing an unintended pregnancy exceed the additional drug costs (compared to LNG $1.5 \mathrm{mg}$ ).

UK, and ulipristal acetate (UPA) (ellaOne ${ }^{\circledR}$ ), a new method of EHC that can be taken up to 120 hours after UPSI. The new method has demonstrated greater efficacy ${ }^{11}$ yet costs more. We examine whether it is worth paying more to avoid an additional unintended pregnancy.

\section{Methods}

This analysis compares two methods of EHC in women who present after having UPSI. The reference drug is LNG $1.5 \mathrm{mg} 1.5 \mathrm{mg}$, which is compared to $30 \mathrm{mg}$ UPA. The analysis is conducted from $0-120$ hours after UPSI and pregnancy events are followed until they end in induced abortion, miscarriage or childbirth. No consideration is given to future episodes of UPSI or subsequent pregnancy events. In the base case all women presenting for EHC up to 120 hours after UPSI are considered for analysis.

The perspective taken is that of the NHS and therefore only direct health costs are considered in the analysis. These direct health care costs include cost of appointments with the general practitioner (GP) and midwife, procedural costs of ultrasound scans, hospital costs of miscarriage, induced abortion and delivery. There are many other costs associated with unintended pregnancy that have not been included in this analysis: indirect costs such as patient transport to appointments, time off work, and costs of providing for a baby; and intangible costs such as the emotional impact of having an unintended pregnancy (whether ending in abortion, miscarriage or childbirth).

The total costs of each treatment and the resulting costs 


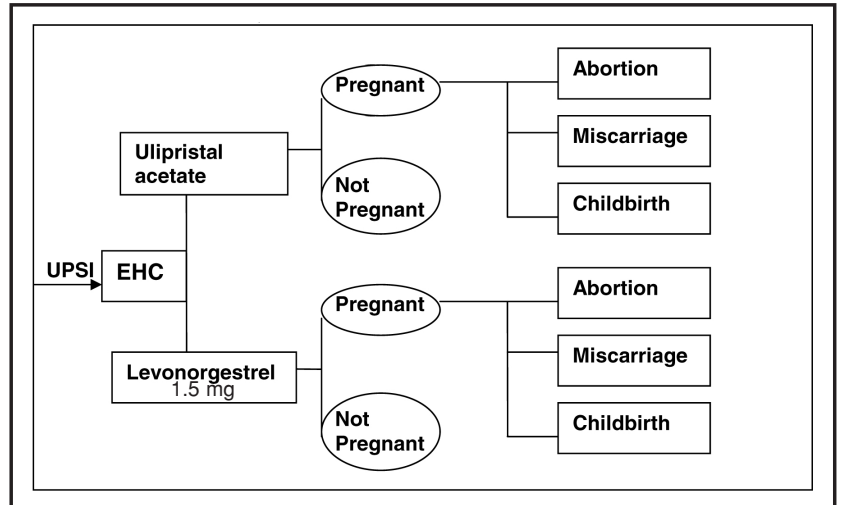

Figure 1 Decision tree model of emergency hormonal contraception (EHC) following unprotected sexual intercourse (UPSI)

of unintended pregnancy from using either treatment are calculated. The difference in the total costs is divided by the additional efficacy of UPA to provide an incremental costeffectiveness ratio (ICER). This is the amount of additional money that must be spent to avoid one more unintended pregnancy with UPA than with LNG $1.5 \mathrm{mg}$.

Figure 1 illustrates the decision tree model used to compare UPA to LNG $1.5 \mathrm{mg}$ in EHC. Women taking EHC may subsequently become pregnant or not. If they become pregnant they may decide to have an abortion, or elect to continue to delivery or the pregnancy may miscarry.

The average costs of induced abortion, miscarriage or childbirth used in this analysis were derived from The National Schedule of Reference Costs 2008 ( $£ 672, £ 474$ and $£ 1578$ for abortion, miscarriage and delivery respectively $)^{8}$ and comprise the cost of induced abortion, miscarriage or childbirth weighted according to whether they were carried out as an inpatient (elective and non-elective; short and long stay), day case or outpatient. The cost of an induced abortion was weighted according to whether it was surgically or medically managed based on the 2008 data that $62 \%$ of all induced abortions are surgically managed. 6 The cost of miscarriage included only those women requiring hospital treatment; no cost was included for women who may present to their GP or in the primary care setting. The costs of childbirth were weighted according to the method of delivery in 2007-2008 12 (i.e. 63\% normal delivery, $25 \%$ Caesarean section and $12 \%$ assisted delivery).

However, the cost of a childbirth is greater than the hospital cost of the delivery. Therefore an estimate was made of the total direct health care costs of childbirth including antenatal and postnatal care. The National Institute for Health and Clinical Excellence (NICE) Routine Antenatal Care for Healthy Women ${ }^{13}$ was used as a basis for calculating antenatal care costs and the NICE Routine Postnatal Care of Women and their Babies ${ }^{14}$ was used as a basis for calculating routine postnatal care costs. As such, these cost estimates are the most conservative given that a proportion of women may experience complications. Furthermore, women who decide to continue with a pregnancy despite having taken active steps to prevent it through seeking EHC may require additional support. The cost of antenatal care includes eight midwife appointments (first initial appointment at home or clinic, six subsequent community visits, one appointment at maternity unit), two ultrasound scans (one dating at 10-14 weeks and one anomaly scan at 18-20 weeks), two blood tests (one at booking and one at 28 weeks). The cost of optional tests such as the triple test or nuchal fold scan have not been included. The cost of postnatal care comprises the care during the 6 weeks following delivery and includes three home visits by a midwife, one home visit by a health visitor, a newborn hearing test and a 6-8-week check for both mother and baby by a GP. No costs for the neonatal period in hospital or any complications arising in that period are included. All these cost elements of a pregnancy resulting in delivery are shown in Table 1.

Table 1 Cost of a pregnancy resulting in childbirth

\begin{tabular}{llll}
\hline & Number & Unit cost & Total cost \\
\hline Antenatal care & & & \\
Midwife appointments & & $£ 77$ & $£ 77$ \\
$\quad$ Booking (home/community) & 1 & $£ 41$ & $£ 246$ \\
Ongoing (community) & 6 & $£ 46$ & $£ 46$ \\
$\quad$ Final (hospital) & 1 & $£ 53$ and £68 & $£ 121$ \\
Ultrasound & 2 & & $£ 6$ \\
Blood test & 2 & $£ 51$ & $£ 153$ \\
Postnatal care & 3 & $£ 51$ & $£ 51$ \\
Midwife visits & 1 & $£ 30$ & $£ 30$ \\
Health visitor & 1 & & $£ 72$ \\
Neonatal hearing test & 2 & & $£ 802$ \\
6-8 week check GP (1 mother/1 baby) & & & $£ 1578$ \\
Community costs & & & $£ 2380$ \\
Hospital costs & & &
\end{tabular}

aSource: National Institute for Health and Clinical Excellence Clinical Guidelines 37 and 62.13,14 bSource: The National Schedule of Reference Costs 2008-09.8 GP, general practitioner.

Table 2 Costs and probability of pregnancy outcomes following emergency hormonal contraceptiona

\begin{tabular}{llll}
\hline Pregnancy outcome & Probabilitya & Cost & Probability x cost \\
\hline Induced abortion & 0.66 & $£ 672$ & $£ 444$ \\
Miscarriage & 0.16 & $£ 474$ & $£ 76$ \\
Delivery & 0.18 & $£ 2380$ & $£ 428$ \\
Unintended pregnancyb & 1.0 & - & $£ 948$ \\
\hline
\end{tabular}

aSource: Comparative clinical trial data (HRA Pharma, data on file, June 2010).

bThis is calculated by adding all the figures in the column above. 
Table 3 Drug-specific costs and probability of pregnancy following emergency hormonal contraception

\begin{tabular}{lll}
\hline & Ulipristal acetate & Levonorgestrel 1.5 mg \\
\hline Drug costs & $£ 16.95$ & $£ 5.37$ \\
Consultation for EHC with GPb & $£ 36.00$ & $£ 36.00$ \\
Probability of pregnancyc 0-120 hours after UPSI & $1.28 \%$ & $2.20 \%$ \\
Probability of pregnancy 0-72 hours after UPSIc (first sensitivity analysis) & $1.36 \%$ & $2.16 \%$ \\
\hline
\end{tabular}

aSource: British National Formulary. 27

bSource: Unit Costs of Health and Social Care 2008.26

cSource: Comparative clinical trial data. ${ }^{11.15}$

EHC, emergency hormonal contraception; GP, general practitioner; UPSI, unprotected sexual intercourse.

Looking specifically at women who become pregnant following EHC in the clinical trials (HRA Pharma, data on file, June 2010) the percentage of unintended pregnancies that end in delivery is $18 \%$, induced abortion $66 \%$ and miscarriage $16 \%$. Applying these percentages to the average cost of a delivery ( $£ 2380)$, induced abortion ( $£ 672)$ and miscarriage (£474) gives an average cost of an unintended pregnancy of $£ 948$. This calculation of unintended pregnancy used in the model is outlined in Table 2.

This economic analysis was conducted using directly comparative data from the clinical development of UPA, which are regarded as the best evidence. No other data sources were sought since these are the only comparative data for UPA and LNG $1.5 \mathrm{mg}$ and were conducted amongst UK women and so pertinent for this analysis. The probability of becoming pregnant after taking UPA or LNG $1.5 \mathrm{mg}$ is based on the clinical trial data from the metaanalysis of both comparative trials and is shown in Table $3^{11,15}$ together with the costs for each EHC treatment strategy. Given that in this meta-analysis there were no pregnancies in the UPA group in women presenting $>72$ hours after UPSI, analysis was not performed on the 72-120-hour period. However, results were calculated for the 0-72-hour period in sensitivity analysis as this represents the licensed timeframe of the comparator product LNG $1.5 \mathrm{mg}$. As there was no difference in adverse event rate between the two treatment arms, ${ }^{15}$ the cost of adverse events was not included in the model.

\section{Statistical analysis: sensitivity analysis}

We performed a number of sensitivity analyses to test the robustness of the results. This involved changing the values for which there was a degree of uncertainty. In the first sensitivity analysis we examined the impact on the results of using the pregnancy rates over a different time frame by using costs from the base case along with the pregnancy rates for 0-72 hours after UPSI as outlined in Table 3.

In the second sensitivity analysis we looked at the impact of the cost of an unintended pregnancy on our results. In the UK, overall pregnancy outcomes (i.e. planned and unintended pregnancies) have a much greater proportion of women delivering $(75 \%)$ and subsequently fewer induced abortions (20\%) and miscarriages that require hospital treatment $(5 \%)^{6,12,16}$ than those observed in the clinical trials of EHC. When calculated using these percentages, an average cost of a pregnancy (whether planned or not) is $£ 1858$. However, as this cost is for all pregnancies it is likely to be an overestimation of the cost of unintended pregnancy where a greater proportion of women will have an induced abortion. Two studies in Scotland looking at contraceptive use in unintended pregnancy have shown that approximately $90 \%$ of induced abortions, almost $8 \%$ of deliveries and $12 \%$ of miscarriages are a result of unintended pregnancy. 5,17 If these proportions are applied to the numbers of maternity events recorded in the UK in 2008, the proportion of women whose unintended pregnancy ends in induced abortion is $73 \%$, with $24 \%$ delivering and $3 \%$ miscarrying. If these proportions are applied, the average cost of an unintended pregnancy in the UK is estimated to be $£ 1076$. This cost is presented in the sensitivity analysis to illustrate the impact that varying the outcomes of an unintended pregnancy has on the results.

Finally we present a combination of both sensitivity analyses, by taking the pregnancy rate at 0-72 hours and using the cost of applying the outcomes of an unintended pregnancy in the UK to determine whether UPA would remain cost-effective using these estimates.

\section{Results}

The results of the base case are presented in Table 4. The costs for each treatment strategy are shown together with the difference in pregnancy rate and the corresponding incremental cost effectiveness ratio (ICER). This represents the cost of preventing one additional unintended pregnancy. In other words, how much does the NHS have to pay to prevent one more unintended pregnancy with UPA? The NHS will spend less on the cost of unintended pregnancies using UPA rather than LNG $1.5 \mathrm{mg}$ because there are fewer unintended pregnancies with UPA. However, this does not outweigh the additional drug administration costs that are

Table 4 Base case results

\begin{tabular}{lll}
\hline $\mathbf{0 - 1 2 0}$ hours after UPSI & Ulipristal acetate & Levonorgestrel $\mathbf{1 . 5} \mathbf{~ m g}$ \\
\hline Cost of unintended pregnancya & $£ 12.13$ & $£ 20.85$ \\
Drug administration costs & $£ 52.95$ & $£ 41.37$ \\
Total costsc & $£ 65.08$ & $£ 62.22$ \\
Difference in pregnancy rated & $£ 311.00$ & $0.92 \%$ \\
ICERe & \\
\hline
\end{tabular}

aProbability of pregnancy in given timeframe multiplied by the cost of unintended pregnancy (for ulipristal acetate $=1.28 \% \times £ 948$ and for levonorgestrel $1.5 \mathrm{mg} 2.2 \% \times £ 948$ ).

bCost of general practitioner consultation plus cost of drug.

cEquals $a+b$.

dDifference in pregnancy rate in given timeframe taken from clinical trial data. ${ }^{11,15}$

e Calculated from difference in total costs divided by difference in pregnancy rate (£65.08-£62.22)/0.92\%.

ICER, incremental cost-effectiveness ratio; UPSI, unprotected sexual intercourse. 
Table 5 Sensitivity analyses results

\begin{tabular}{|c|c|c|c|c|c|c|}
\hline Parameter & \multicolumn{2}{|c|}{$\begin{array}{l}\text { First sensitivity } \\
\text { analysis }\end{array}$} & \multicolumn{2}{|c|}{$\begin{array}{l}\text { Second sensitivity } \\
\text { analysis }\end{array}$} & \multicolumn{2}{|c|}{$\begin{array}{l}\text { Sensitivity analyses } \\
\text { combined }\end{array}$} \\
\hline Time post-UPSI & 0-72 hours & & $0-120$ hours & & 0-72 hours & \\
\hline EHC method & UPA & LNG $1.5 \mathrm{mg}$ & UPA & LNG 1.5 mg & UPA & LNG $1.5 \mathrm{mg}$ \\
\hline Cost of unintended pregnancya & $£ 12.89$ & $£ 20.47$ & $£ 13.77$ & $£ 23.67$ & $£ 14.63$ & $£ 23.24$ \\
\hline Drug administration costs ${ }^{b}$ & $£ 52.95$ & $£ 41.37$ & $£ 52.95$ & $£ 41.37$ & $£ 52.95$ & $£ 41.37$ \\
\hline Total costsc & $£ 65.84$ & $£ 61.84$ & $£ 66.72$ & $£ 65.04$ & $£ 67.58$ & $£ 64.61$ \\
\hline Difference in total costs ${ }^{d}$ & $+£ 4.00$ & & $+£ 1.68$ & & $+£ 2.97$ & \\
\hline Difference in pregnancy rate & $0.80 \%$ & & $0.92 \%$ & & $0.80 \%$ & \\
\hline ICER $^{\dagger}$ & $£ 500.00$ & & $£ 183.00$ & & $£ 372.00$ & \\
\hline
\end{tabular}

aProbability of pregnancy in given timeframe multiplied by the cost of unintended pregnancy.

bCost of general practitioner consultation plus cost of drug.

cEquals $a+b$.

dDifference between total costs of UPA and LNG $1.5 \mathrm{mg}$.

eDifference in pregnancy rate in given timeframe taken from clinical trial data. ${ }^{11,15}$

fDifference in total costs divided by difference in pregnancy rate.

EHC, emergency hormonal contraception; ICER, incremental cost-effectiveness ratio; LNG 1.5 mg, levonorgestrel 1.5 mg; UPA, ulipristal acetate; UPSI, unprotected sexual intercourse.

incurred with UPA compared to LNG $1.5 \mathrm{mg}$. Taking all these costs into consideration, the cost of preventing one additional unintended pregnancy with UPA is £311. This is the amount that the NHS has to pay to use UPA in order to prevent one additional pregnancy, thus avoiding the cost of an unintended pregnancy (£948).

Table 5 shows the results of the sensitivity analyses. In the first sensitivity analysis, when the pregnancy rates for 0-72 hours are used instead of 0-120 hours as in the base case, the cost of preventing one additional unintended pregnancy with UPA is $£ 500$. This is still less than the cost of the unintended pregnancy (£948).

In the second sensitivity analysis the cost of an unintended pregnancy is based on UK outcomes of unintended pregnancy rather than those observed in the EHC clinical trials. In this scenario the cost of preventing one additional unintended pregnancy with UPA is $£ 183$. When combining both scenarios (i.e. combining estimates from the clinical trial at $<72$ hours and UK pregnancy outcomes data), the cost of preventing one additional unintended pregnancy is $£ 372$. All these calculations are illustrated in Table 5 in more detail.

\section{Discussion}

This study showed that the additional cost to the NHS of providing UPA instead of LNG $1.5 \mathrm{mg}$ was less than the cost of an unintended pregnancy. Furthermore, the ICER for preventing one pregnancy was much less than the cost of abortion, miscarriage or delivery, whether taken within 120 hours or 72 hours of UPSI. Given that by using UPA the NHS will have to pay for fewer unintended pregnancies, we conclude that UPA is a cost-effective alternative to LNG $1.5 \mathrm{mg}$.

A statistically significant difference in pregnancy rate is seen between UPA and LNG $1.5 \mathrm{mg}$ when taken 0-120 hours after UPSI and is the most appropriate comparison for the base case analysis. ${ }^{11}$ As LNG $1.5 \mathrm{mg}$ is not licensed for use $>72$ hours after UPSI and its efficacy diminishes beyond 72 hours after UPSI, ${ }^{18,19}$ UPA may be particularly useful for this population. However, as there were no pregnancies observed in this population in the UPA group in the comparative trial this does not allow a realistic comparison to be made between treatment groups. On the basis of the current data UPA would cost less to prevent more pregnancies. However, as only $10 \%$ of women presented in this time frame in the clinical trials it is insufficient to present a robust analysis for the post-72 hours UPSI population. The sensitivity analysis therefore presents data for the 0-72 hours post-UPSI population, where there is also a statistically significant difference in pregnancy rate. ${ }^{11}$
Data on pregnancy outcomes were taken from the clinical trials (for the base case) and included delivery, induced abortion or miscarriage. In clinical practice a small proportion of pregnancies are ectopic. Use of contraception decreases the risk of pregnancy and therefore ectopic pregnancy. According to the UK Medical Eligibility Criteria for Contraceptive Use (UKMEC) there does not appear to be an increased risk of ectopic pregnancy following use of progestogen-only methods of EC. ${ }^{20}$ However, no data on ectopic pregnancy rates following EHC were identified (such a rare outcome is not detected in clinical trials) and therefore this outcome was omitted in both treatment arms of the model.

Costs of delivery are based on hospital deliveries only; the cost of home births was not included. The rate of home births in UK was $2.68 \%$ in 2007 including women who were subsequently transferred to hospital. ${ }^{21}$ Costs of home births would also include community maternity services but a valid estimate of these was not identified. This omission exists in both treatment arms of the model.

The rate of miscarriage used in the model is lower than the generally accepted rate quoted in clinical practice of $20-25 \% .22$ This is because a proportion of pregnancies that end in very early miscarriage would not necessitate hospital admission or therefore cost. The sensitivity analysis addresses this by presenting data when the rate of miscarriage reflects those treated in hospital (from $16 \%$ in base case to $3 \%$ in sensitivity analysis). This latter rate is the number of unintended pregnancies that result in miscarriage and require hospital treatment, based on the number of miscarriages that are treated in hospitals and the proportion of these that are unintended.

The cost of neonatal care following delivery was not included in the model and this omission exists in both arms. There is no evidence to suggest adverse birth outcomes following EHC failure so no adjustment was made for this. Overall the direct cost estimates for unintended pregnancy are conservative and in practice may be higher. Such estimates would tend to lead to lower ICERs for UPA in practice.

No indirect costs or intangible costs were included in our analysis since the perspective taken was that of the NHS. However, the impact of having an unintended pregnancy, whatever the outcome, is not insignificant. Indirect costs could include time off work to attend appointments, travelling costs to appointments for the women and the direct costs of bringing up a child, which will also include social cost implications. Intangible costs may include the emotional impact of having an unintended pregnancy. 
No economic assessments of EHC use in the UK have been published. UK Health Technology Assessment organisations such as the Scottish Medicines Consortium (SMC) and NICE have reviewed regular methods of contraception, including the combined hormonal vaginal ring, IUD and hormonal implant. The ICER for the vaginal ring compared with the weighted average of other contraceptive methods was estimated to be $£ 3337$ per pregnancy avoided. ${ }^{23}$ According to the NICE Long-acting Reversible Contraception (LARC) guideline, ${ }^{24}$ the ICER of the IUD versus the injectable in the first year of use was £339. The ICER of the implant (most effective LARC) versus IUD (least costly among LARC) dropped from $£ 17866$ in the second year of use to $£ 1403$ in Year 15 of use. However, any comparison of ICERs of EC with routine contraception should be made with caution as the target population is different and both treatment compliance and discontinuation have a high impact on the cost-effectiveness of routine contraception. Furthermore, EC is used in an 'acute' situation to prevent pregnancy after UPSI whereas routine contraception is used over a longer time period and therefore the ICERs presented relate to a different timeframe. EHC is used to prevent pregnancy in a woman who has identified herself 'at risk' of pregnancy following exposure through UPSI. These women are not comparable with women who are defined 'at risk' of pregnancy due to their fertility status and lack of contraceptive use. Finally, women who use regular contraception may also require EC due to incorrect use (e.g. missed pills).

In clinical practice women are often offered the IUD as a method of EC. However, fewer than $1 \%$ of women presenting for EC say they select this option. ${ }^{25}$ Even in community contraception clinics where there are more likely to be personnel trained to fit the IUD, the uptake is only $3 \% .9$ Whilst the IUD would confer ongoing contraceptive benefits, these can only be realised if it is seen as acceptable by women presenting for EC. The cost-effectiveness of the IUD would be affected by the rate of discontinuation of the IUD.

UPA is a cost-effective alternative to LNG $1.5 \mathrm{mg}$ for all women presenting for EC after UPSI. The ICER of UPA compared to LNG $1.5 \mathrm{mg}$ is estimated at $£ 311$ in the base case (ranging from $£ 183$ to $£ 500$ in sensitivity analysis). The cost-effectiveness of UPA is maintained as time postUPSI progresses. As the cost of avoiding one additional pregnancy (ICER) is less than the cost of an unintended pregnancy or the cost of an induced abortion, we conclude that it is worth paying the additional cost of UPA.

\section{Acknowledgements}

The authors wish to thank Anna Glasier and Martyn Walling for their helpful comments.

\section{Statements on funding and competing interests}

Funding This study was funded by HRA Pharma (UK) Ltd.

Competing interests Ramona Schmid is employed by HRA Pharma.

\section{References}

1 McGuire A, Hughes D, The cost-effectiveness of family planning service provision. J Public Health Med 1996; 18: 189-196.

2 Trussell J, Wiebe E, Shochet T, Guilbert E. Cost savings from emergency contraceptive pills in Canada. Obstet Gynecol 2001; 97: 789-793.

3 Trussell $\mathrm{J}$, Calabretto $\mathrm{H}$. Cost savings from use of emergency contraceptive pills in Australia. Aust N Z J Obstet Gynaecol 2005; 45: 308-311.

4 Trussell J, Koenig J, Ellertson C, Stewart F. Preventing unintended pregnancy: the cost-effectiveness of three methods of emergency contraception. Am J Public Health 1997; 87: 932-937.

5 Lakha F, Glasier A. Unintended pregnancy and use of emergency contraception among a large cohort of women attending for antenatal care of abortion in Scotland. Lancet 2006; 368: 1782-1787.

6 Department of Health. Statistical Bulletin. Abortion Statistics, England and Wales: 2008. 2009. http://www.dh.gov.uk/ prod consum dh/groups/dh digitalassets/documents/digitalass et/dh_099714.pdf [Accessed 5 July 2010].

7 Final induced abortion of pregnancy KCl Chapter: Data for 2008. May 2009. http://www.isdscotland.org/isd/information-andstatistics.jsp?pContent|D $=1918 \& \mathrm{p}$ applic $=\mathrm{CCC} \& \mathrm{p}$ service $=$ Con tent.show\&\#Activity in 2005 [Accessed 5 July 2010]

8 Department of Health. The National Schedule of Reference Costs 2008-09. 2009. http://www.dh.gov.uk/en/Publications andstatistics/Publications/PublicationsPolicyAndGuidance/DH_0 98945 [Accessed 5 July 2010].

9 Statistical Services Contraception 1997-2008 The NHS Information Centre KT31 return. http://www.ic.nhs.uk/webfiles/ publications/Contrceng\%202006-07/Statistical\%20Tables\%20 Contraception\%20services\%202006-07.xls [Accessed 5 July 2010].

10 Intrauterine devices and intrauterine systems. ESHRE Capri Workshop Group. Hum Reprod Update 2008; 14: 197-208.

11 Glasier AF, Cameron ST, Fine PM, Logan SJ, Casale W, Van Horn $\mathrm{J}$, et al. Ulipristal acetate versus levonorgestrel for emergency contraception: a randomised non-inferiority trial and meta-analysis. Lancet 2010; 375(9714): 555-562.

12 The Health and Social Care Information Centre. Hospital Episode Statistics Table 32: Method of delivery by NHS trust and site, 2008-09. 2009. http://www.hesonline.nhs.uk/ Ease/servlet/ ContentServer?sitel $\mathrm{D}=1937$ \&category $\mid \mathrm{D}=1060$ [Accessed 5 July 2010].

13 National Institute for Health and Clinical Excellence (NICE). Antenatal Care: Routine Care for the Healthy Pregnant Women (Clinical Guideline No. 62). 2008. http://www.nice.org.uk/ nicemedia/pdf/CG062NICEguideline.pdf [Accessed 5 July 2010].

14 National Institute for Health and Clinical Excellence (NICE). Routine Postnatal Care of Women and Their Babies (Clinical Guideline No. 37). July 2006. http://www.nice.org.uk/nice media/pdf/CG37NICEguideline.pdf [Accessed 5 July 2010].

15 Creinin MD, Schlaff W, Archer DF, Wan L, Frezieres R, Thomas $\mathrm{M}$, et al. Progesterone receptor modulator for emergency contraception: a randomized controlled trial. Obstet Gynecol 2006; 108: 1089-1097.

16 The Health and Social Care Information Centre. Hospital Episode Statistics Figure 2: Miscarriage and ectopic pregnancies that resulted in a hospital stay; rates per 100 deliveries, 1997-98 to 2007-08. 2009. http://www.hesonline. nhs.uk/Ease/servlet/ ContentServer?sitel $\mathrm{D}=1937$ \& category $\mid \mathrm{D}=1063$ [Accessed 5 July 2010].

17 Schünmann C, Glasier A. Measuring pregnancy intention and its relationship with contraceptive use among women undergoing therapeutic abortion. Contraception 2006; 73: 520-524.

18 von Hertzen H, Piaggio G, Van Look PF. Emergency contraception with levonorgestrel or the Yuzpe regimen. Task Force on Postovulatory Methods of Fertility Regulation. Lancet 1998; 352(9144): 428-433.

19 von Hertzen H, Piaggio G, Ding J, Chen J, Song S, Bártfai G, et al; WHO Research Group on Post-ovulatory Methods of Fertility Regulation. Low dose mifepristone and two regimens of levonorgestrel for emergency contraception: a WHO multicentre randomised trial. Lancet 2002; 360: 1803-1810.

20 Faculty of Family Planning and Reproductive Health Care Clinical Effectiveness Unit. UK Medical Eligibility Criteria for Contraceptive Use (UKMEC 2009). 2009. http://www.fsrh.org/ admin/uploads/UKMEC2009.pdf [Accessed 5 July 2010].

21 BirthChoice UK. Non-Institution Birth Rates for UK. http://www.birthchoiceuk.com/Frame.htm [Accessed 5 July 2010].

22 Royal College of Obstetrics and Gynaecology. Early Miscarriage: Information for You. January 2008. http://www.rcog.org.uk/ womens-health/clinical-guidance/early-miscarriage-informationyou [Accessed 5 July 2010].

23 Scottish Medicines Consortium (SMC) Advice. $11.7 \mathrm{mg}$ etonogestrel/2.7 mg ethinyl estradiol vaginal ring (NuvaRing $($ ) (No. 502/08). September 2009. http://www.scottishmedicines. org.uk/files/11.7mg\%20etonogestrel\%202.7mg\%20\%20ethinyle stradiol\%20vaginal\%20ring\%20NuvaRing.pdf [Accessed 5 July 2010]

24 National Institute for Health and Clinical Excellence (NICE). Long-acting Reversible Contraception: The Effective and Appropriate Use of Long-acting Reversible Contraception (Clinical Guideline CG30). October 2005. http://www.nice. org.uk/nicemedia/pdf/CG03Ofullguideline.pdf [Accessed 5 July 2010]

25 Lader D. Office for National Statistics. Opinions Survey Report No. 41. Contraception and Sexual Health 2008/09. 2009. http://www.statistics.gov.uk/downloads/theme_health/contra 2008-9.pdf [Accessed 5 July 2010].

26 Curtis L. Personal Social Services Research Unit University of Kent. Unit Costs of Health and Social Care 2008. 2008. http://www.pssru.ac.uk/pdf/uc/uc2008/uc2008.pdf [Accessed 5 July 2010].

27 British National Formulary. September 2009. www.bnf.org [Accessed 5 July 2010]. 